Recent Advances in Communication, Electronics \& Electrical Engineering

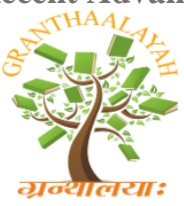

INTERNATIONAL JOURNAL OF RESEARCH -

RACEEE - 17

\title{
RECENT ADVANCES IN OPTICAL NETWORK TECHNOLOGIES AND ITS ROLE IN TELECOMMUNICATION INDUSTRY
}

\author{
Bimlendu Shahi ${ }^{* 1}$, Divya Jha ${ }^{2}$, Dr. Dushyanth N D ${ }^{3}$, Dr. Suresh $\mathrm{D}^{4}$ \\ $* 1,2,3,4$ Department of Electronics and Communication Engineering, School of Engineering and \\ Technology, Jain University, Bangalore-562112, India
}

DOI: https://doi.org/10.29121/granthaalayah.v5.i4RACEEE.2017.3317

\begin{abstract}
Optical networking technology has been advancing swiftly for some decades now. The high bandwidth and low attenuation features of fiber optics has made it the major element of telecommunication infrastructure. To achieve the ever-growing demand of data in telecommunication industry, researchers have explored various networking technologies in optical domain as well as advanced equipment and programmability, boosting transmission up to gigabit and beyond. This paper reviews various optical communication technologies, recent advances in optical network, it's role in telecommunication industry and its challenges.
\end{abstract}

Keywords: Optical Network; Telecommunication; Bandwidth.

Cite This Article: Bimlendu Shahi, Divya Jha, Dr. Dushyanth N D, and Dr. Suresh D. (2017). "RECENT ADVANCES IN OPTICAL NETWORK TECHNOLOGIES AND ITS ROLE IN TELECOMMUNICATION INDUSTRY." International Journal of Research Granthaalayah, 5(4) RACEEE, 15-22. 10.29121/granthaalayah.v5.i4RACEEE.2017.3317.

\section{Introduction}

The global intercommunications network has pervaded and has turned into an essential necessity of everyday life, empowering telecommunication industry at Customer Service Provider to provide innumerable services. Optical links which support enormous bandwidth has developed as one the efficient data and information transmission channel that can serve the need of modern society of conveying huge amount of data over longer distances. The race for ever enrichment in measure of performance for transportation network can prolong and extend limit of a usability of single fiber has been further enhanced upto several Gb/s in 1990 to further more increment upto $\mathrm{Tb} / \mathrm{s}$ today [2]. The huge parallel interconnects for the support of its connectivity information exchange among the data center servers and supercomputers leads to advancement of Optical Network. 


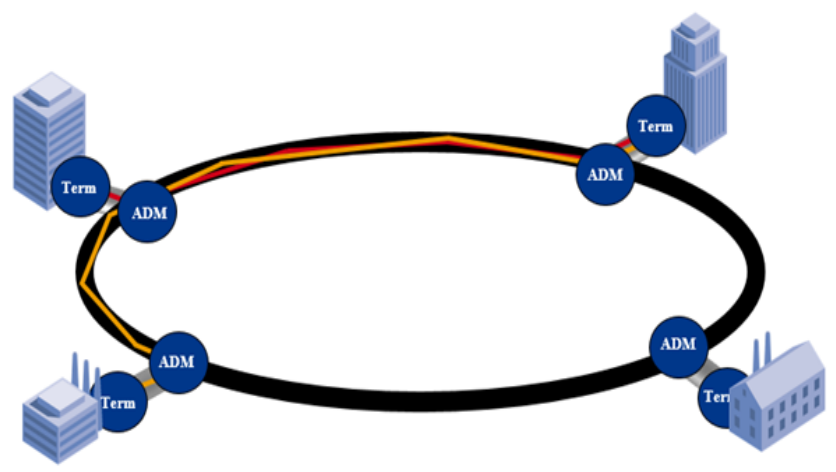

Figure 1: Optical Network topology.

Optical communication is a wide and vase research field and swiftly changing research domain, where researchers around the globe and different expertise from different fields such as electronics, communications, photonics, signal processing have provided their contribution side by side to meet the ever-increasing demands for higher capacity, lowering energy consumption and its cost in system design to grant novel applications and emerging in new technologies in Optical domain. The fig 1 shows the optical network topology having an Add Drop Mux (ADM), in which two high speed signal are terminated and the low speed signals they contain are removed.

In recent years, we have seen the development of more proficient optical transport capabilities conveyed by the optical equipment and its framework providers as ultra-fast optical and server to explore systems extended internationally. This advancement has been achieved by the always quickening improvement of fast interconnects and optical transceivers for data-center servers. Recent advances in optical communication increment the limits of correspondence framework as well as enhance the framework dynamicity and survivability [10]. Different new and upcoming advanced technologies are created to build the data transmission of individual wavelength channels and the quantity of wavelengths transmitted per fiber. Different new and upcoming optical technologies have additionally been produced to strengthen different developing applications, including new and upcoming, on-request and high demand data rate applications, in an adaptable, power efficient and cost-efficient way.

\section{Recent Advances}

The development of recent innovations to setup access networks by using optical network technologies has been considered as one of the best choice for long-haul topology. The applications of different electronic layers, extensive limit per channel, adaptable wavelength (frequency) allocation, and quick optical switching and equipment handling can bring the innovative advancement in the optical networking technologies. Recently, different vendors around the globe are able to make possible by carrying huge bundles of Internet packet data and Ethernet frames or SONET/SDH frame over single fiber using wavelength division multiplexing technique [7]. Also, researchers are going to make possible more further enhancement in capacity, scalability and manageability of optical networks by employing advancement in equipment and building its efficient programmability, advanced coding, and modulation 
Recent Advances in Communication, Electronics \& Electrical Engineering techniques. Multi-core parallel DSP processor embedded routers play significant role in switching networks, which also limits the required constraint for the minimum power consumption required per bit switched and in other hand need to expand number of packets and its frame with its pace.

C'edric Ware et al. [3] have discussed the limitations with the optics which has no adaptable technique for buffering bundles of packets (without which the switching is defenseless against dispute); or decoding and handling their headers (to choose where to send them). These both issues are aggravated by asynchronous arrival of packets. The authors have thus expressed doubt as to whether OPS can help with this excessive energy consumption. Since, By placing ROADM in current OCS networks builds more transparency to modulation and consumes minimum energy than all other electronic switching technique particularly for traffic in which all channels can be switched without requirement of kind of sub-wavelength granularity. And still, after all that, OCS stays semi static practically speaking: endeavors to make it dynamic come up not just against issues of between channel interactions because of optical non-linearity and particularly control power dynamism in optical amplifiers; additionally, the way that routing and wavelength/spectrum task is a NP-hard [18] issue, consequently hard to tackle continuously against changing network conditions. Variety of these developments makes it too early to tell exactly how OPS will be used in next-generation networks. Thus, author concluded that Optical packet switching (OPS) is able to control the drain in power, by utilizing low-energy optics cost per bit transmitted and usability of minimum conversion of optical-to-electronic (O-E) conversions [3].

Jun He et. al. [10] discussed about optical components, such as large port count switches and low cost ROADM, which are developed to satisfy new requirements in data center communications. With the electronic transmission reaching its limit, data centers are likely to benefit from use of optics to carry data. Lam et al. [20] reviewed several shortcomings and requirement of network transformation of optical technologies and in other its growing trends so that for efficient management of server data-centers. As indicated by [20], server data-center needs to utilize full spectrum of fiber optic technologies deployed in the network from short reach of deployed network to long haul deployed network areas. For example, Due to mechanism of compensation in dispersion and its advancement in signal modulation results in glooming of next generation intra-datacenter. Farrington et al. [18] also presented a framework called as Helios which utilizes the equipment of optical/electrical in hybrid form, which causes reduces in cost of cable and number of switching element for deploying the network and in other hand reduces the consumption of power to provide trade-off for deployment of network. Wang et al. [17] also demonstrated c-Through as a prototype which explores a efficient design methodology of $\mathrm{HyPaC}$. As the demonstrated prototype provides compatibility with existing server data-center networks to deliver efficient de-multiplexing and demand estimation of growing traffic upto end host joined in the network.

H. Yang et al. [22] presented a new software-defined data center optical interconnection (SUDOI) architecture to enable extensive user access from the perspective of multi-layer networking modes. The feasibility of framework are compared with respect to rate of resource allocation and its blocking probability in network setup. Authors have also experimentally demonstrated efficiency of the proposed architecture on an optical-as-a-service, which is testbed 
Recent Advances in Communication, Electronics \& Electrical Engineering

using Open- Flow-enabled optical nodes. The functional modules of SUDOI architecture, including the core elements of various controllers are described.

Schares L et al. [19] studied the upcoming advancement into datacenter systems due to new optical technologies and discussed about several network topology and its optical network architectures. Authors have focused basically on software controlled optical circuit switch, which is applicable for optically attached memory systems to permit able low latency streaming for data-driven workloads. Rofoee et al. [21] reported on a user/application-driven multi technology data-plane for optical sub-wavelength network and also for reservation of available network resources which are based on an extended GMPLS-Path Computation Engine Sub-wavelength Assignment Engine. As shown in [22], a very low latency data delivery is achieved by the enhanced control plane in testbeds, which also demonstrated flexible connectivity patterns with various bit rates. This flexibility of different technologies for sub-wavelength spectrum is due to nature of statistical multiplexing.

There has been a huge research to move to all-optical network to increase scalability and operational flexibility. The introduction of Wavelength-selective-switch (WSS) is suitable for 80 -wavelength systems. The utilization of optical technologies based on Liquid Crystal on-Silicon (LCOS) array supports upto $1 \mathrm{GHz}$ very fine spectrum and hence allow wavelength selective switches and Fast ROADMs to select wide-range of add-drop spectral shapes and make it more flexible.

Along with development in hardware, there has been discovery of new technologies to make network scalable as well as cost-effective. R. Sanchez et al. [14] discussed about the comparative study which deals with respect to a economic and technical aspects about four different optical standard technologies for NG-PON standard: GPON, XGPON, WDM-PON, and the emerging TWDMPON. In this contribution, the authors analyzed NG-PON technologies to provide target temporal guarantee and as well as delivery of $1 \mathrm{~Gb} / \mathrm{s}$ symmetrical bandwidth at minimum cost to local subscribers. K. Y. Cho et al. [12] have demonstrated a long-reach wavelength-divisionmultiplexed passive optical network (WDM PON), which uses efficient digital coherent receivers deployed in the network setup and use of reflective Semiconductor Optical Amplifier (RSOA). Similarly, Patrick P et al. have discussed various PON technologies which are capable of supporting data rates more than $10 \mathrm{~GB} / \mathrm{s}$ such as TDM, Colorless WDM, Optical OFDM, CDM, Hybrid PON.

Optics can also be used for on-chip communications to achieve space, power and spectrally efficiency. Previously, OEO technology faces several issues like area, efficiency, power consumption and also in reliability due to assembly of discrete components. However, Infinera Corporation brought core Optical back-haul network technologies using PIC based technology for execution of OEO Vision. PIC based technology is able to miniaturize and integrate several number of WDM technologies monolithically on a chip in the year 2005. The applications of MEMS with PIC technology might be able to build more efficient and scalable optical system to correct the switching at several network elements. One of these technologies which gives the replacement of fixed transponder is tunable transponder. Tunable transponder can be tuned to any of the available wavelengths by receiving or generating only single wavelength at a sytstem. 
Recent Advances in Communication, Electronics \& Electrical Engineering This mechanism simplifies several operations of network topology by remotely assigning one wavelength to a connection of transponder.

\section{Role of Optical Network Technologies in Telecommunication}

Optical transport technologies allow to deliver bandwidth that meets the needs of large enterprises, cloud operators, and internet content providers as illustrated in fig 2 . They give network capacity, efficiency, and flexibility which supports services that can be deployed and reconfigured on demand. Due to its capability to deliver more wavelength capacity and wavelengths per fiber in optics, telecommunication industry is able to deliver the services upto Gbps and more for each user end.

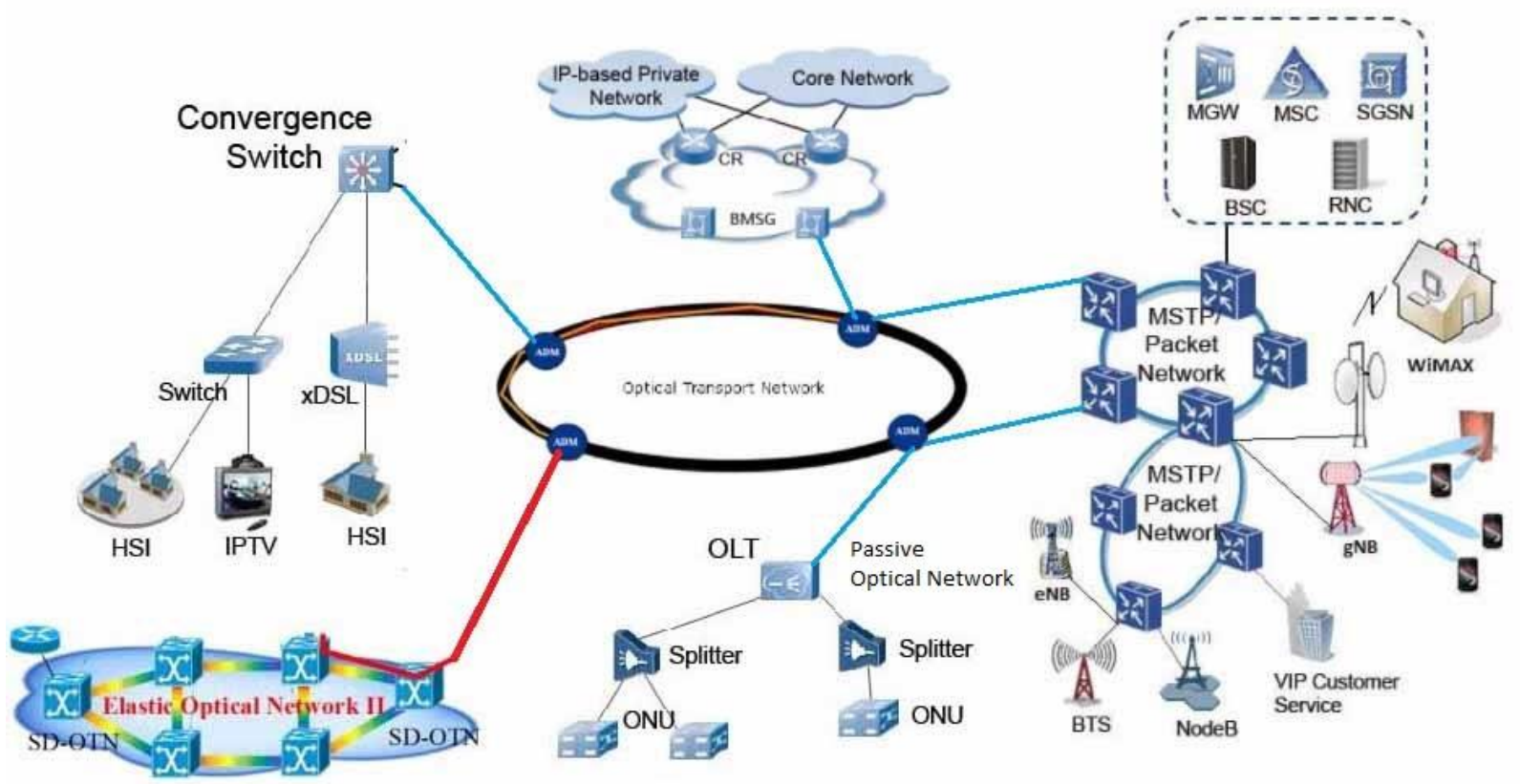

Figure 2: Deployment of Optical Network technologies in Telecommunication Industry.

Mobile traffic volumes are expected to increase exponentially by 2020 and beyond. The evolution of LTE to LTE-Advanced with VoLTE and 5G will increasingly demand higher capacity and lower latency capabilities from operator networks where tight coordination is key for efficient spectrum utilization. Operators face the most challenges to meet these requirements in metropolitan and dense urban areas, where footprint is critical and therefore new alternative optical network technologies are needed with high spectral efficiency at a lower cost.

At some point, all the Customer Service Providers around the globe are upgrading their bandwidth capability to grant the non-stoppable growing demand for voice call, on demand video services and also cloud-based services over two copper using DSL applications. CSP are able to provide these services effectively due to low-attenuation and ultra-bandwidth capability at back-haul network through convergence switch as illustrating in fig 2. CSP over the metro convergence need to maintain low TCO i.e. total cost of ownership over single unified billing and management system for all services granted to end-user. There is requirement to grant all the permitted services utilization at same time ensuring there is no interruption or any kind of 
Recent Advances in Communication, Electronics \& Electrical Engineering disturbances to any other services. ADSL i.e Asynchronous Digital Subscriber Line must be scalable and robust with efficient performance capability to grant the multimedia services over the converged network topology. CSP need to collaborate and modernize existing and upcoming technology effectively to a single optical packet network to have strong contribution towards the digital world.

\section{Challenges}

Different technologies are being introduced for enhancement of optical networking system. Different service providers are adopting different technologies considering the trade-off in different technologies and their requirement. The interaction among sub-networks with different technologies has made the network complex than ever. There are challenges in maintaining and securing the network. However, there are challenges in each of the technology which should overcome for proper utilization of the technology in telecommunication industry.

There might occur critical issues in preserving the same splitting ratio and coverage in case of upgradation of bandwidth more than $\mathrm{Gb} / \mathrm{s}$ while evolving over next generation technologies due to deployment of TDM PON [5]. Similarly, in case of WDM PON, big challenge is reducing complexity of transmission in upstream and also its several expenses in maintenance due to its complexity in transmission [13]. OFDM systems were experimentally exhibited in the R\&D laboratory as a Long-haul network having high bandwidth with fast processing DSP but still this exhibited system has not been commercialized in the market for sale [5].

One of the major challenges all-optical networks is facing is constraint in terms of wavelength continuity. This may arise due to a scenario in blocking connection of lightpath due to unavailability of wavelength on all link of path. But, still there are all the links available with sufficient bandwidth capacity. Also, there might be other challenges occurred due to susceptibility of transient nature in optical amplifiers. These transient natures appear in the optical amplifier due to sudden variance and toggling up and down in Level of power over the transmission line of fiber leads to error bursts. Next big challenges is to maintain and further control the overall processing power required to distinguish the signals in the various cores or modes, and realizing optical amplifiers and ROADMs in order to prolong full coverage that operate simultaneously on all of the signals in the fiber for an all-optical solution, [8].

One of the major challenges related to SDM systems is to guarantee low crosstalk over extensive optically networked communication distances, with integrated amplifiers, transponders, connectors, splices, SDM fibers, and NEs such as spectral and spatial cross-connects [12]. In recent years, use of hollow core fiber has been proposed. However, there is a challenge of manufacturing fiber of few nm extending over $100 \mathrm{kms}$ of length and yet minimize loss windows [12]. Some of the challenges in elastic optical networks include establishing a physical transmission design methodology for heterogeneous DWDM signals, elastic transponders and regenerators with reasonable photonic integration, and management and control plane technology compatible with emerging software defined network (SDN) [14].

Another major challenge common to all the optical networking technologies is managing the faults as the network size grows exponentially [10]. Blocking situations may emerge because of 
Recent Advances in Communication, Electronics \& Electrical Engineering numerous requests of data at same time resulting in fault and is difficult to avoid this scenario by operator.

\section{Conclusion}

Optical Network has grown as the only transport technology which can fulfill the enormous data demand at high rate. Different technologies, advanced hardware and chips with programmability and software development have contributed to the advancement of Optical Networking in terms of capacity, speed, scalability and security. However, demand for high speed communication is ever increasing which has led the researchers to explore further possibilities to fulfill the traffic demand. This paper reviewed different optical networking technologies, recent advances in optical networks and some of their challenges. Use of different optical networking technologies based on the cost, demand and availability of resources can prove to be beneficial. Further development of high speed DSPs at the cost which can be commercialized can enhance the network capacity. With the development of network that can accommodate ever increasing traffic demand and its wide deployment, it increases the number of network elements, which gives rise to the challenge of managing the network. Along with the advancement in technologies, enhancement in management and control plane can make the upcoming new technologies feasible.

\section{References}

[1] Tianhua Xu, Nikita A. Shevchenko,Domaniç Lavery, Daniel Semrau,Gabriele Liga, Alex Alvarado, Robert I. Killey, and Polina Bayvel," Modulation format dependence of digital nonlinearity compensation performance in optical fibre communication systems", OPTICS EXPRESS 3311,Vol. 25, No. 4 ,20 Feb 2017.

[2] Erik Agrell et al.," Roadmap of optical communications", Journal of Optics, 2016, doi:10.1088/2040-8978/18/6/063002.

[3] C'edric Ware, Wiem Samoud, Philippe Gravey and Mounia Lourdiane," Recent advances in optical and hybrid packet switching", ICTON (IEEE)2016.

[4] Pierluigi Poggiolini," Recent Advances in Non-linear Fiber Propagation Modeling", OSA 2016.

[5] Patrick P. Iannone and Kenneth C. Reichmann," Optical Access Beyond $10 \mathrm{~Gb} / \mathrm{s}$ PON",IEEE,2010.

[6] Y. C. Chung," Recent Advancement in WDM PON Technology", ECOC Technical Digest,OSA,2011.

[7] Admela Jukan and Joe Mambretti," Evolution of Optical Networking Toward Rich DigitalMedia Services", Proceedings of the IEEE, Vol.100, No. 4, April 2012.

[8] Adel A. M. Saleh and Jane M. Simmons," All-Optical Networking V Evolution, Benefits, Challenges, and Future Vision", Proceedings of the IEEE, Vol. 100 No. 5, May 2012.

[9] Peter J. Winzer, "Optical Networking Beyond WDM", IEEE Photonics Journal, DOI: 10.1109/JPHOT.2012.2189379,2012.

[10] Jun He et al.," A survey on recent advances in optical communications", Elseveir,2013.Charles Laperle and Maurice O'Sullivan," Advances in High-Speed DACs, ADCs, and DSP for Optical Coherent Transceivers", JOURNAL OF LIGHTWAVE TECHNOLOGY, VOL. 32, NO. 4, FEBRUARY 15, 2014.

[11] D. J. Richardson, J. M. Fini and L. E. Nelson," Space-division multiplexing in optical fibres", NATURE PHOTONICS, VOL 7, MAY 2013. 
Recent Advances in Communication, Electronics \& Electrical Engineering

[12] K. Y. Cho, U. H. Hong,S. P. Jung,Y. Takushima, A. Agata, T. Sano, Y. Horiuchi,M. Suzuki, and Y. C. Chung," Long-reach 10-Gb/s RSOA-based WDM PON employing QPSK signal and coherent receiver", OPTICS EXPRESS 15353, Vol. 20, No. 14, 2 July 2012.

[13] Masahiko Jinno," Recent Advances in Elastic Optical Networks", International Conference on Photonics in Switching, 2013.

[14] Rafael Sánchez, José Alberto Hernández, Julio Montalvo García, and David Larrabeiti," Provisioning $1 \mathrm{~Gb} / \mathrm{s}$ Symmetrical Services with Next-Generation Passive Optical Network Technologies", IEEE Communications Magazine, February 2016.

[15] O. Gerstel, et al., IEEE Commun. Mag., 50, S12-S20, 2012.

[16] S. Gringeri, B. Basch, V. Shukla, R. Egorov, and T. J. Xia, BFlexible architectures for optical transport nodes and networks, [ IEEE Commun. Mag., vol. 48, no. 7, pp. 40-50, Jul. 2010.

[17] Y. Wang, X. Cao, and Q. Hu, "Routing and spectrum allocation in spectrum-sliced elastic optical path networks," in IEEE International Conference on Communications (ICC), Jun. 2011.

[18] Farrington Nathan, Porter George, Radhakrishnan Sivasankar, Bazzaz Hamid Hajabdolali, Subramanya Vikram, Fainman Yeshaiahu, et al. Helios: a hybrid electrical/optical switch architecture for modular data centers. In: Proceedings of the ACM SIGCOMM 2010 conference (SIGCOMM '10), New York, NY, USA; 2010. p. 339-50.

[19] Schares L, Kuchta DM, Benner AF. Optics in future data center networks. In: 2010 IEEE 18th annual symposium on high performance interconnects (HOTI), 18-20 August, 2010. p. 104, 108.

[20] Lam CF, Hong Liu, Koley B, Xiaoxue Zhao, Kamalov V, Gill, et al. Fiber optic communication technologies: what's needed for datacenter network operations. IEEE Commun Mag 2010;48(7):32,39.

[21] Rofoee B, Zervas G, Yan Y, Simeonidou D, Bernini G, Carrozzo G, et al. Demonstration of low latency intra/inter data-centre heterogeneous optical subwavelength network using extended GMPLS-PCE control-plane. Opt Express 2013;21:5463-74.

[22] Wang Guohui, Andersen David G, Kaminsky Michael, Papagiannaki Konstantina, Ng TS Eugene, Kozuch Michael, Ryan Michael. c-Through: part-time optics in data centers. In: Proceedings of the ACM SIGCOMM 2010 conference (SIGCOMM '10), New York, NY, USA; 2010. p. 327-38.

[23] Fields M, Foley J, Kaneshiro R, McColloch L, Meadowcroft D, Miller F, et al. Transceivers and optical engines for computer and datacenter interconnects. In: Optical fiber communication conference, paper OTuP1.

*Corresponding author.

E-mail address: bimlendu_yo@yahoo.com 